\title{
Collective effects in the collapse-revival phenomenon and squeezing in the Dicke model
}

\author{
G. Ramon, $\mathrm{E}$ C. Brif, \\ Department of Physics, Technion - Israel Institute of Technology, Haifa 32000, Israel
}

\begin{abstract}
Resonant interaction of a collection of two-level atoms with a single-mode coherent cavity field is considered in the framework of the Dicke model. We focus on the role of collective atomic effects in the phenomenon of collapses and revivals of the Rabi oscillations. It is shown that the behavior of the system strongly depends on the initial atomic state. In the case of the initial half-excited Dicke state we account for a number of interesting phenomena. The correlations between the atoms result in a suppression of the revival amplitude, and the revival time is halved, compared to the uncorrelated fullyexcited and ground states. The phenomenon of squeezing of the radiation field in the atom-field interaction is also discussed. For the initial fully-excited and ground atomic states, the field is squeezed on the short-time scale, and squeezing can be enhanced by increasing the number of atoms. Some empirical formulas are found which describe the behavior of the system in excellent agreement with numerical results. For the half-excited Dicke state, the field can be strongly squeezed on the long-time scale in the case of two atoms. This kind of squeezing is enhanced by increasing the intensity of the initial coherent field and is of the same nature as revival-time squeezing in the Jaynes-Cummings model. The appearance of this long-time squeezing can be explained using the factorization approximation for semiclassical atomic states.
\end{abstract}

42.50.Ct, 42.50.Md, 42.50.Dv, 32.80.-t

\section{INTRODUCTION}

Since the pioneering work of Dicke [1] on cooperative spontaneous emission, a great deal of attention has been devoted to the interaction of the radiation field with a collection of two-level atoms located within a distance much smaller than the wavelength of the radiation. Such a system is commonly referred to as the Dicke model (for a review see, e.g., Ref. [2]). A particular case of the Dicke model, when atoms interact with a single-mode radiation field inside a cavity, was considered by Tavis and Cummings [3]. The Tavis-Cummings Hamiltonian is mathematically equivalent to the trilinear boson Hamiltonian describing various nonlinear optical processes [ $[$ ]. The single-atom version, known as the Jaynes-Cummings model [5], is the simplest and one of the most popular models of quantum optics. In spite of its simplicity, the Jaynes-Cummings model shows a variety of interesting nonclassical phenomena such as vacuum-field Rabi oscillations, sub-Poissonian photon statistics, and squeezing of the radiation field (for reviews see, e.g., Refs. [6.7]).

One of the most interesting quantum features of the Jaynes-Cummings model is the phenomenon of collapses and revivals of the Rabi oscillations, which manifests itself in the clearest way when the cavity field is prepared initially in the coherent state [8]. The shape of collapses and revivals is determined by the initial photon-number distribution. A similar behavior can be found also in the many-atom case [9 11]. For a sufficiently strong coherent field, the nonlinearity in the Rabi frequency is slight and the system exhibits regular dynamics in the form of collapses and revivals of the oscillations. However, in the many-atom case there exist anharmonic collective corrections which modify the shape of the collapses and revivals related to the photon-distribution mechanism [12]. In the present work we study in detail these collective effects for different initial atomic states. If atoms are prepared initially in the ground state or in the fullyexcited state, then the system behaves rather similarly to the single-atom case, although collective effects manifest themselves clearly in the length of the revival time. But, the results are different when the atoms are prepared initially in the half-excited Dicke state. This state is well known as the superradiant atomic state in the context of collective spontaneous emission in open space [1.2. 2. It was also found [13] that the half-excited Dicke state strongly modifies the resonance fluorescence spectrum of the atomic system. However, to the best of our knowledge, this state was rarely considered in the context of the Tavis-Cummings (cavity) version of the Dicke model. When the atoms are prepared in the half-excited Dicke state the system exhibits a number of interesting phenomena. In particular, the amplitude of the Rabi oscillations is strongly suppressed and relative intensities of revivals are essentially changed. This suppresion of the revival amplitude is similar to the trapping phenomenon occuring in the case of a single atom prepared in the equally weighted superposition of the two levels 114.

It has been known for a long time [15, 16] that the nonlinear character of the Jaynes-Cummings model leads to squeezing in one of the quadratures of an initially coherent cavity field. It was also predicted [17 that strong squeezing can be obtained in the Jaynes-Cummings model near the revival times for large initial intensities of the field. This phenomenon of strong revival-time squeezing was explained in Ref. [18] using the factorization approximation for the so-called semiclassical atomic states [19]. Butler and Drummond [20] showed that short-time squeezing can be enhanced in the Dicke model compared to the single-atom case. Seke 21] considered field and atomic squeezing in the Dicke model without the rotating-wave approximation and in the presence of losses. Analytical approaches to squeezing in the strongfield limit 22] and in the weak-field limit 23] were pre- 
sented. Higher-order squeezing in the Dicke model was studied in Ref. 24]. For atoms prepared initially in the ground state or in the fully-excited state, the uncertainty of the field quadrature rapidly oscillates and squeezing occurs during short periods of time at the very beginning of the time evolution. On the long-time scale the temporal behavior of the quadrature uncertainty is correlated with times of collapses and revivals of the Rabi oscillations. On the other hand, for the initial half-excited Dicke state, the behavior of squeezing is qualitatively different. The quadrature uncertainty oscillates on the long-time scale, with a period of the order of the revival half-time, and strong squeezing is obtained in the case of two atoms. The value of squeezing is enhanced by the intensity of the initial coherent field. This phenomenon is of the same nature as revival-time squeezing in the Jaynes-Cummings model and can be explained using the factorization approximation for the semiclassical atomic states. In general, squeezing is better and its duration is much longer for the initial two-atom half-excited Dicke state than for the ground and fully-excited states.

\section{THE MODEL AND METHODS OF SOLUTION}

We consider the resonant interaction between $N$ twolevel atoms and the single-mode radiation field inside a lossless cavity. In the rotating-wave approximation, the Tavis-Cummings interaction Hamiltonian reads $(\hbar=1)$ :

$$
H=g\left(a^{\dagger} J_{-}+a J_{+}\right)
$$

Here and in the following we use the interaction picture. In Eq. (2.1) $g$ is the coupling constant, $a$ and $a^{\dagger}$ are the annihilation and creation operators of the field mode, $J_{+}$ and $J_{-}$are the collective atomic raising and lowering operators. They satisfy the $\mathrm{su}(2)$ Lie algebra,

$$
\left[J_{+}, J_{-}\right]=2 J_{z}, \quad\left[J_{z}, J_{ \pm}\right]= \pm J_{ \pm},
$$

where $J_{z}$ is the operator of atomic inversion. In terms of the standard Pauli matrices, describing each two-level atom, one obtains

$$
J_{ \pm}=\frac{1}{2} \sum_{i=1}^{N} \sigma_{ \pm}^{(i)}, \quad J_{z}=\frac{1}{2} \sum_{i=1}^{N} \sigma_{z}^{(i)} .
$$

If the Schwinger realization of the $\mathrm{SU}(2)$ generators is used, the Hamiltonian (2.1) becomes the trilinear boson Hamiltonian describing nonlinear optical processes such as parametric conversion and Raman and Brillouin scattering [4]. An interesting physical realization of the Hamiltonian (2.1) is given by the coupling of the internal levels of atoms or ions to a mode of their quantized oscillatory motion in a harmonic trap 25].

The total excitation operator

$$
L=a^{\dagger} a+J_{z}+N / 2
$$

commutes with the Hamiltonian (2.1) and is an integral of motion. Another integral of motion is the $\mathrm{SU}(2)$ Casimir operator $\mathbf{J}^{2}=J_{z}^{2}+\frac{1}{2}\left(J_{+} J_{-}+J_{-} J_{+}\right)$. We describe the state of the atomic system in terms of the $\mathrm{SU}(2)$ orthonormal basis $|j, m\rangle_{\text {at }}(m=j, j-1, \ldots,-j)$,

$$
\begin{aligned}
J_{z}|j, m\rangle_{\text {at }} & =m|j, m\rangle_{\text {at }}, \\
\mathbf{J}^{2}|j, m\rangle_{\text {at }} & =j(j+1)|j, m\rangle_{\text {at }} .
\end{aligned}
$$

In the context of the atomic realization (2.3), the states $|j, m\rangle$ at are the symmetric Dicke states:

$$
|j, m\rangle_{\text {at }}=\left(\begin{array}{c}
N \\
p
\end{array}\right)^{-1 / 2} \sum \prod_{k=1}^{p}|+\rangle_{l_{k}} \prod_{l \neq l_{k}}|-\rangle_{l},
$$

where $|+\rangle_{l}$ and $|-\rangle_{l}$ are the upper and lower states, respectively, of the $l$ th atom, and the summation is over all possible permutations of $N$ atoms. If only symmetric atomic states are considered, then the "cooperative number' $j$ is equal to $N / 2$ and $p=m+j$ is just the number of excited atoms.

The Hilbert space $\mathcal{H}$ of the atom-field system can be decomposed into a direct sum of finite-dimensional invariant subspaces $\mathcal{H}_{L}$ :

$$
\mathcal{H}=\bigoplus_{L=0}^{\infty} \mathcal{H}_{L}
$$

Each invariant subspace $\mathcal{H}_{L}$ is spanned by the orthonormal basis $|n\rangle_{\mathrm{f}}|j, L-j-n\rangle_{\text {at }}$, where $|n\rangle_{\mathrm{f}}$ are the Fock states of the radiation field, $a^{\dagger} a|n\rangle_{\mathrm{f}}=n|n\rangle_{\mathrm{f}}$. For $L<N$, $n=0,1, \ldots, L$ and $\operatorname{dim}\left(\mathcal{H}_{L}\right)=L+1$; for $L \geq N$, $n=L-N, L-N+1, \ldots, L$ and $\operatorname{dim}\left(\mathcal{H}_{L}\right)=N+1$. If the field is initially in the Fock state $\left|n_{0}\right\rangle_{\mathrm{f}}$ and the atoms are in the Dicke state $\left|j, m_{0}\right\rangle_{\text {at }}$, the state of the system will evolve in the invariant subspace $\mathcal{H}_{L}$ with $L=n_{0}+m_{0}+j$. For the field and/or atoms prepared initially in a superposition state, one should take into account contributions from different subspaces.

The exact solution of the problem is obtained by the diagonalization of the interaction Hamiltonian (2.1) in each of the invariant subspaces $\mathcal{H}_{L}$ involved 3. It is known [3, 4 that in the basis $|n\rangle_{\mathrm{f}}|j, L-j-n\rangle_{\text {at }}$ the Hamiltonian is given by a tridiagonal matrix with symmetric eigenvalues and the corresponding characteristic equation can be reduced to an algebraic equation of order $\left[\operatorname{dim}\left(\mathcal{H}_{L}\right) / 2\right]$. Therefore, an analytical solution is possible when only invariant subspaces with $\operatorname{dim}\left(\mathcal{H}_{L}\right) \leq 9$ are involved. However, already for $\operatorname{dim}\left(\mathcal{H}_{L}\right)>3$, analytical solutions are rather complicated 26 28. Note that for $\operatorname{dim}\left(\mathcal{H}_{L}\right) \geq 4$ the eigenvalues are not equidistant, so the time evolution is not periodic even within a single subspace. Semiclassical approximate solutions were proposed 29 31 which give the time evolution of systems governed by a trilinear Hamiltonian of type (2.1) in terms of elliptical functions. A perturbative analytical approach to the problem with weak fields was developed by Kozierowski and co-workers 
[27,28, 32, 33]. In the present work we use the exact solution based on the numerical diagonalization of the interaction Hamiltonian (2.1).

\section{COLLAPSES AND REVIVALS}

We study the temporal behavior of the atom-field system in the Dicke model for the cavity field prepared initially in the coherent state $|\alpha\rangle_{\mathrm{f}}$ :

$$
|\alpha\rangle_{\mathrm{f}}=e^{-|\alpha|^{2} / 2} \sum_{n=0}^{\infty} \frac{\alpha^{n}}{\sqrt{n !}}|n\rangle_{\mathrm{f}} .
$$

Without loss of generality we consider only real values of $\alpha$. The initial atomic state is supposed to be one of the Dicke states $|j, m\rangle_{\text {at }}$ (recall that $j=N / 2$ ). Two possibilities which are frequently considered in the literature are the fully-excited state $|j, j\rangle_{\text {at }}$ and the ground state $|j,-j\rangle_{\text {at }}$. We are also interested in the half-excited Dicke state $|j, 0\rangle_{\text {at }}$.

Since the coherent field state is a superposition of many Fock states $|n\rangle_{\mathrm{f}}$, the invariant subspaces $\mathcal{H}_{L}$ with different values of $L=n+m+j$ contribute to the evolution. The temporal behavior of the atomic inversion $\left\langle J_{z}\right\rangle$ is given by the sum of the appropriately weighted atomic responses to each Fock state. (The mean photon number $\left\langle a^{\dagger} a\right\rangle$ is connected with the atomic inversion by the fact that $\langle L\rangle=\left\langle a^{\dagger} a\right\rangle+\left\langle J_{z}\right\rangle+N / 2$ is a constant.) In the resonant Jaynes-Cummings model $(N=1)$ with initially unexcited $\left(m=-\frac{1}{2}\right)$ or excited $\left(m=\frac{1}{2}\right)$ atom, one obtains [8]

$$
\left\langle J_{z}\right\rangle=m \sum_{n=0}^{\infty} P_{n} \cos \Omega_{n, m} \tau,
$$

where $\tau=g t$ is the scaled time,

$$
\Omega_{n, m}=2 \sqrt{n+m+1 / 2}
$$

is the scaled Rabi frequency corresponding to each subspace, and $P_{n}$ is the photon-number distribution. For the initial coherent state, $P_{n}=\exp (-\bar{n}) \bar{n}^{n} / n$ ! is the Poissonian distribution and $\bar{n}=|\alpha|^{2}$ is the initial mean photon number. Then, due to the property of this distribution, the most regular dynamics occurs for large values of the initial mean photon number $\bar{n}$. Contributions corresponding to different $n$ 's interfere in such a manner that they initially go out of phase, after that acquire a common phase, and this process is repeated resulting in a series of collapses and revivals, as shown in Fig. I1 (a). The revival time $\tau_{R}$ can be estimated using the condition [8, 34

$$
\tau_{R}\left(\Omega_{\bar{n}+1, m}-\Omega_{\bar{n}, m}\right)=2 \pi,
$$

which gives $\tau_{R}=2 \pi \sqrt{\bar{n}+m+1 / 2}$.
One should expect a similar behavior also for the Dicke model in the strong-field domain $\bar{n}>N$ [9 12]. Of course, the collapses and revivals related to the photonnumber distribution would be modified by the collective atomic effects due to the fact that the eigenvalues of the interaction Hamiltonian are not equidistant. In the strong-field limit $\bar{n} \gg N$ the anharmonic corrections to the eigenvalues become small, and one should expect a quite regular behavior similar to that of the resonant Jaynes-Cummings model. However, we will see that there exist reasons for an irregular behavior that are much more important than just the anharmonicity of the eigenvalues. In fact, the initial atomic state determines how important will be various factors leading to irregularities in the behavior of the system.
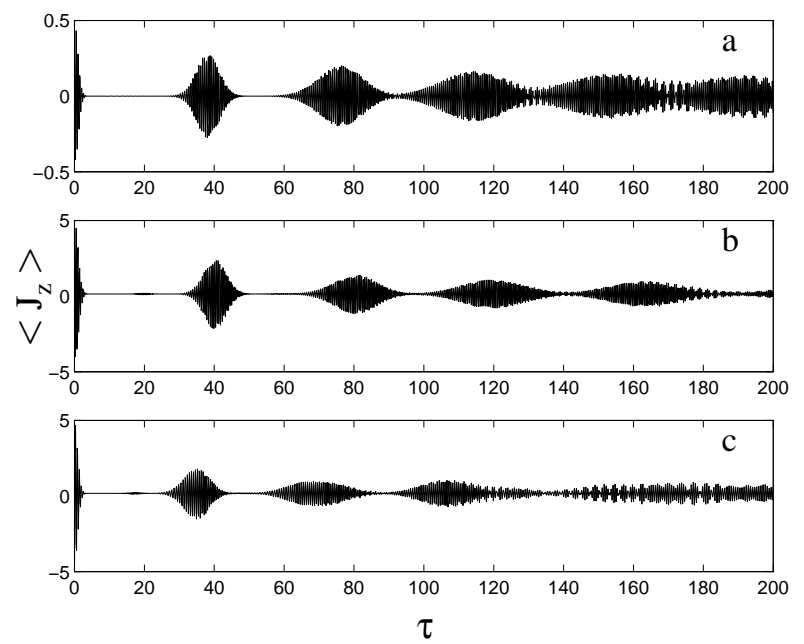

FIG. 1. The atomic inversion $\left\langle J_{z}\right\rangle$ versus the scaled time $\tau=g t$ for the initial coherent field state with $\bar{n}=36$ interacting with (a) one excited atom (the Jaynes-Cummings model) and with 10 atoms prepared in (b) the fully-excited state $|j, j\rangle_{\text {at }}$ and (c) the ground state $|j,-j\rangle_{\text {at }}$.

We first consider the cases with the atoms prepared initially in the fully-excited and ground states. The temporal behavior of the atomic inversion $\left\langle J_{z}\right\rangle$ is shown in Fig. 11(b,c) for the case of $N=10$ and $\bar{n}=36$, with atoms prepared in (b) the fully-excited state $|j, j\rangle_{\text {at }}$ and (c) the ground state $|j,-j\rangle_{\text {at }}$. The first feature to note is the dependence of the revival time on the initial atomic state. Similarly to the Jaynes-Cummings model, we can estimate the revival time for the initial atomic state $|j, m\rangle_{\text {at }}$ as

$$
\tau_{R}=2 \pi \sqrt{\bar{n}+m+1 / 2},
$$

where we use the strong-field limit expression for the Rabi frequency (i.e., neglect the anharmonic corrections to the eigenvalues). Formula (3.5) is in a good agreement with our numerical results, as demonstrated in Fig. 2. The difference between the revival times for the fully-excited state and the ground state is particularly obvious when $N$ is not too small compared to $\bar{n}$. The second feature is 
that the spread of the revivals is determined only by the photon statistics of the initial field state [34] and does not depend on the value of $m$. These two observations may explain why the temporal behavior loses its regularity for the initial ground state notably faster than for the initial fully-excited state. Indeed, we see in Fig. 1(b) four regular revivals for $m=j$, while in Fig. 11(c) for $m=-j$ the behavior starts to be irregular already at the end of the third revival. The reason is that the spread of the revivals increases with time and neighboring revivals start to overlap, which leads to the loss of regularity. Since for $m=-j$ the revival time is shorter than for $m=$ $j$, the overlapping of the revivals and the corresponding irregularity occur earlier.
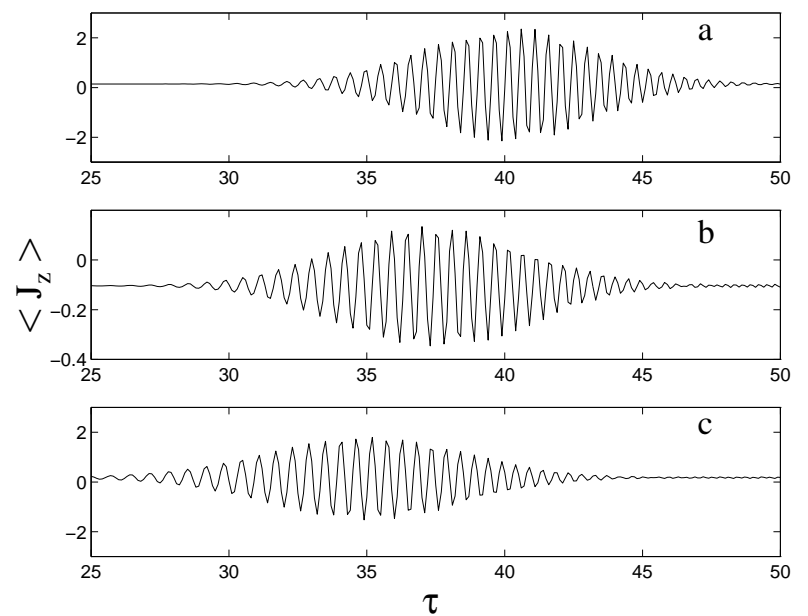

FIG. 2. The atomic inversion $\left\langle J_{z}\right\rangle$ versus the scaled time $\tau=g t$ for the initial coherent field state with $\bar{n}=36$ interacting with with 10 atoms prepared in (a) the fully-excited state $|j, j\rangle_{\text {at }}$, (b) the half-excited Dicke state $|j, 0\rangle_{\text {at }}$, and (c) the ground state $|j,-j\rangle_{\text {at }}$. The region of the first revival is shown, demonstrating the dependence of the revival time on the initial atomic state.

Another factor that leads to differences between the behavior of the system in the cases $m=j$ and $m=-j$ is the dependence of the anharmonic corrections to the eigenvalues on the value of $m$. In order to give a representative example, we considered the eigenvalues $h_{i}$ of the interaction Hamiltonian for the subspaces with $L=n+m+j$ for $n=36, N=10,20,30$ and $m= \pm j, 0$. The ratio $f_{51}=h_{5} / h_{1}$ of the 5 th and 1 st eigenvalues was chosen as a parameter representing the anharmonicity (for equidistant eigenvalues $f_{51}$ is exactly 5 ). The numerical results are listed in Table [1]. We see that for given $N$ the value of $f_{51}$ increases with decrease of $m$, i.e., irregular effects related to the anharmonicity are most important for $m=-j$. For $m=j$ the value of $f_{51}$ decreases slightly with increase of $N$, but the overall contribution of the anharmonic corrections increases with $N$ just because there are more eigenvalues. For $m=-j$ the value of $f_{51}$ increases with $N$, so the importance of the anharmonic corrections here increases with $N$ much faster than for $m=j$. In particular, we see that the amplitude of the revivals for $m=-j$ is smaller than for $m=j$, and this effect becomes more pronounced as $N$ increases. For $\bar{n}=36$, as $N$ increases from 2 to 16 , the relative amplitude $A_{1}$ (the difference between the maximum and minimum values of $\left\langle J_{z}\right\rangle$ in the first revival, divided by $N$ ) decreases from 0.5459 to 0.4039 for $m=j$ and from 0.5362 to 0.2112 for $m=-j$. This effect occurs because the anharmonic corrections partially destroy the interference of the oscillating terms. Of course, as the initial mean photon number $\bar{n}$ increases, the behavior of the system becomes more regular.

Now we turn to the case when the atoms are prepared in the half-excited Dicke state $|j, 0\rangle_{\text {at }}$ (for even values of $N)$. The temporal behavior of the atomic inversion $\left\langle J_{z}\right\rangle$ is shown in Fig. 3 for $\bar{n}=36$ and $N=2,6,10$. Here we see a number of interesting phenomena. First of all, the amplitude of the Rabi oscillations is significantly suppressed. For $\bar{n}=36$, as $N$ increases from 2 to 16 , the relative amplitude $A_{0}$ (the difference between the maximum and minimum values of $\left\langle J_{z}\right\rangle$ in the very beginning of the evolution, divided by $N$ ) increases linearly from 0.006 to 0.027 , according to the empirical formula

$$
A_{0}=0.0015(N+2) \text {. }
$$

For comparison, $A_{0}$ is about 0.9 for $m=j$ and about 0.95 for $m=-j$, being almost independent of $N$.
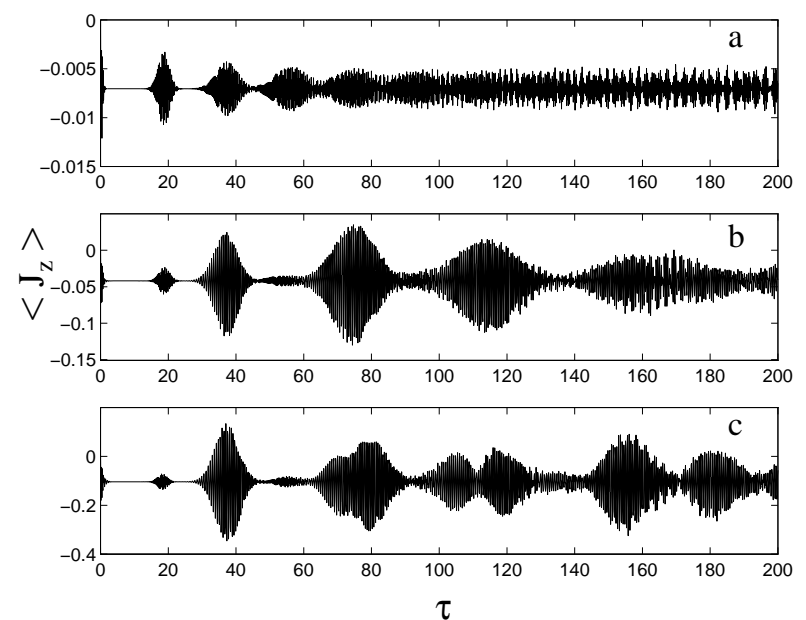

FIG. 3. The atomic inversion $\left\langle J_{z}\right\rangle$ versus the scaled time $\tau=g t$ for the initial coherent field state with $\bar{n}=36$ interacting with $N$ atoms prepared in the half-excited state $|j, 0\rangle_{\text {at }}$ : (a) $N=2$, (b) $N=6$, (c) $N=10$.

Another important feature is the appearance of halftime revivals, i.e., for $m=0$ the revival time is actually $\tau_{R} / 2$. In fact, tiny half-time revivals appear also for $m= \pm j$, but their amplitudes are much smaller than the amplitudes of the first revivals at $\tau_{R}$. As $N$ increases from 2 to 16, the relative amplitude $A_{1 / 2}$ (the difference between the maximum and minimum values of $\left\langle J_{z}\right\rangle$ in the half-time revival, divided by $N)$ increases monotonically from 0.0018 to 0.0171 for $m=j$ and from 0.0019 to 
0.0354 for $m=-j$. The ratio $A_{1 / 2} / A_{1}$ increases in the same range of $N$ from 0.0033 to 0.0424 for $m=j$ and from 0.0035 to 0.1677 for $m=-j$. On the other hand, for $m=0$ the half-time revival and the first revival are, for small values of $N$, of the same order of magnitude. For $N=2$ we even find $A_{1 / 2} / A_{1}>1$. In contradistinction to the cases $m= \pm j$, for $m=0$ the ratio $A_{1 / 2} / A_{1}$ decreases from 1.3427 to 0.0473 as $N$ increases from 2 to 16 . In this range of $N$ the relative amplitude $A_{1 / 2}$ of the half-time revival increases slightly from 0.0037 to 0.0052 , while the relative amplitude $A_{1}$ of the first revival increases much faster: from 0.0028 to 0.1093 . Starting from $N=4$ the amplitude of the first revival exceeds that of the initial oscillations. The ratio $A_{1} / A_{0}$ increases almost linearly from 0.4555 to 4.0476 as $N$ increases from 2 to 16 . The value $\left\langle J_{z}\right\rangle_{C}$ of the atomic inversion during the collapse is always positive for $m= \pm j$. As $N$ increases from 2 to $16,\left\langle J_{z}\right\rangle_{C}$ increases from 0.0036 to 0.3569 for $m=j$ and from 0.0035 to 0.5195 for $m=-j$. On the other hand, for $m=0$ the value of $\left\langle J_{z}\right\rangle_{C}$ is always negative and decreases from -0.0070 to -0.2433 in the same range of $N$. We found that for $m=0$ the value of $\left\langle J_{z}\right\rangle_{C}$ can be well approximated by the empirical formula

$$
\begin{aligned}
& \left\langle J_{z}\right\rangle_{C}=-\left(\frac{N}{k_{1} \alpha-k_{2}}\right)^{z(\alpha)}, \\
& z(\alpha)=k_{3}-\exp \left[-\left(k_{4} \alpha+k_{5}\right)\right],
\end{aligned}
$$

where $k_{1}=7.45, k_{2}=11.16, k_{3}=1.773, k_{4}=0.328$, $k_{5}=1.681$. We also see that in general the dynamics in the case $m=0$ is much more irregular than for $m= \pm j$. All these observations show that the structure of the phenomenon of collapses and revivals is essentially different for the half-excited Dicke state relative to the fully-excited state or the ground state. Inspecting the eigenvalues (see Table (1), we see that the influence of the anharmonic corrections cannot explain this principal difference. As expected, the behavior becomes more regular with increase of the mean photon number $\bar{n}$. For odd numbers of atoms the half-excited Dicke state $|j, 0\rangle_{\text {at }}$ does not exist. However, for sufficiently large odd values of $N$ the Dicke state $\left|j, \frac{1}{2}\right\rangle$ at exhibits properties very similar to those of the half-excited state.

In order to explain the peculiar phenomena discussed above, we consider the exactly solvable case $N=2$. For the fully-excited state $(m=1)$ we find

$$
\begin{aligned}
\left\langle J_{z}\right\rangle= & \sum_{n=0}^{\infty} P_{n} \frac{2}{\Omega_{n, 1}^{4}}\left[(n+3)-(n+1) \cos \left(2 \Omega_{n, 1} \tau\right)\right. \\
& \left.+8(n+1)(n+2) \cos \left(\Omega_{n, 1} \tau\right)\right] .
\end{aligned}
$$

Analogously, we obtain for the ground state $(m=-1)$ :

$$
\begin{aligned}
\left\langle J_{z}\right\rangle= & \sum_{n=0}^{\infty} P_{n} \frac{2}{\Omega_{n,-1}^{4}}\left[(n-2)-n \cos \left(2 \Omega_{n,-1} \tau\right)\right. \\
& \left.-8 n(n-1) \cos \left(\Omega_{n,-1} \tau\right)\right]
\end{aligned}
$$

and for the half-excited Dicke state $(m=0)$ :

$$
\left\langle J_{z}\right\rangle=-\sum_{n=0}^{\infty} P_{n} \frac{1}{\Omega_{n, 0}^{2}}\left[1-\cos \left(2 \Omega_{n, 0} \tau\right)\right] .
$$

Now it is clear why the oscillations are strongly suppressed for $m=0$. Here both the constant and oscillatory terms have the prefactor $P_{n} /(4 n+2)$, while for $m= \pm j$ in addition to the constant and oscillatory terms with prefactors of the order $P_{n} / n$ there exists an oscillatory term with a prefactor of the order $P_{n}$. For $\bar{n}=36$ the suppression is by two orders of magnitude. For $m=0$ the oscillatory term has the frequency $2 \Omega_{n, 0}=2(n+1 / 2)^{1 / 2}$, and the effective revival time is $\tau_{R} / 2=\pi(\bar{n}+1 / 2)^{1 / 2}$. On the other hand, for $m= \pm j$ the leading oscillatory term with the prefactor of the order $P_{n}$ has the frequency $\Omega_{n, \pm 1}$, associated with the revival time $\tau_{R}$, while the smaller oscillatory term with the prefactor of the order $P_{n} / n$ has the double frequency, leading to the half-time revivals. This explains why for $m= \pm j$ the half-time revival is much smaller than the first revival while for $m=0$ both types of revivals are of the same order of magnitude. The more irregular dynamics in the case $m=0$ can be explained by two reasons. First, the nonlinearity of the Rabi frequency $(\sim \sqrt{n})$ is less important for larger values of $n$, so the dynamics is more regular when the main contribution comes from larger $n$ 's. For $m= \pm j$ the prefactors of the leading terms are Poissonian, so for large enough $\bar{n}$, the main contribution will come from the high-frequency terms, resulting in a regular behavior. However, for $m=0$ the prefactors are $P_{n} /(4 n+2)$, so lower frequencies also contribute, which results in a less regular behavior. The second reason is that the revivals start to overlap much earlier if the revival time is halved. The same reason also leads to an additional irregularity in the case $m=-j$ when the number of atoms is relatively large and the half-time revivals are not too small.

The suppression of the revival amplitude for the half-excited Dicke state is similar to the trapping phenomenon which occurs for a single atom prepared in the equally weighted superposition state $2^{-1 / 2}(|+\rangle \pm|-\rangle$ ) (see Ref. 114). In the latter case the population inversion is given by

$$
\begin{aligned}
\left\langle J_{z}\right\rangle= & \frac{1}{2} \sum_{n=0}^{\infty} P_{n}\left[\cos ^{2}(\sqrt{n+1} \tau)\right. \\
& \left.+\frac{\bar{n}}{n+1} \sin ^{2}(\sqrt{n+1} \tau)\right]-\frac{1}{2} .
\end{aligned}
$$

For large $\bar{n}$, the Poissonian distribution is sharply peaked around $\bar{n}$, and the two terms in the sum almost add up to 1 . The remaining oscillating term has the prefactor of the order $P_{n} / n$, so the amplitude of the Rabi oscillations is reduced by the factor of the order $1 / \bar{n}$. As explained above, in such a situation the dynamics is less regular than for the case of initially unexcited or fully excited 
atom. However, the revival time is not halfed for the single atom in the equally weighted superposition state. In the single-atom case the population trapping occurs due to the destructive interference between the contributions of the two levels, while for the two-atom half-excited Dicke state this phenomenon can be explained by the destructive interference between the contributions of the two correlated atoms.

\section{SQUEEZING OF THE RADIATION FIELD}

The coherent radiation field interacting with atoms can acquire interesting nonclassical properties such as sub-Poissonian photon statistics and squeezing. In the present paper we focus on the important quantum phenomenon of squeezing. The initially coherent cavity field can be squeezed when it interacts with a single atom [15,16], and squeezing in the revival-time regime can be very strong for large intensities of the field [17,18]. Butler and Drummond discovered [20] that in the Dicke model collective atomic effects can improve squeezing obtained for short interaction times, compared to the single-atom case. Squeezing in the Dicke model was also considered recently in a number of works 21 23. Here we present a detailed study of squeezing of the initially coherent cavity field, comparing between the ground, fully-excited and half-excited initial atomic states.

The field quadratures, $q=\left(a^{\dagger}+a\right) / \sqrt{2}$ and $p=$ $i\left(a^{\dagger}-a\right) / \sqrt{2}$, satisfy the canonical commutation relation $[q, p]=i$. Then their uncertainties satisfy the Heisenberg relation: $\Delta q \Delta p \geq 1 / 2$, where $(\Delta q)^{2}=\left\langle q^{2}\right\rangle-\langle q\rangle^{2}$ and similarly for $(\Delta p)^{2}$. For the coherent field state (in particular, for the vacuum state) the uncertainties are equal, $\Delta q_{0}=\Delta p_{0}=1 / \sqrt{2}$, and an equality is achieved in the Heisenberg uncertainty relation. A field state is called squeezed, if the uncertainty of one of the quadratures is below the vacuum level, i.e., $\Delta q<1 / \sqrt{2}$ or $\Delta p<1 / \sqrt{2}$. Here we consider the squeezing parameter

$$
\xi=\frac{\Delta q}{\Delta q_{0}}=\sqrt{2} \Delta q
$$

and search for $\xi<1$ that manifests field squeezing.

\section{A. Squeezing for the fully-excited and ground states}

\section{The long-time behavior}

We first consider the temporal behavior of the squeezing parameter $\xi$ on the long-time scale, for the atoms prepared initially in the fully-excited and ground states $(m= \pm j)$.

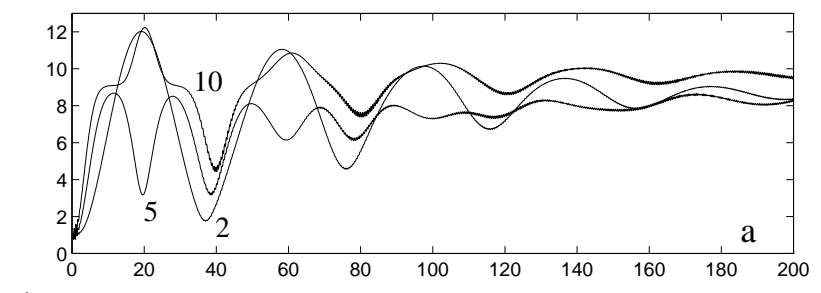

$\xi$

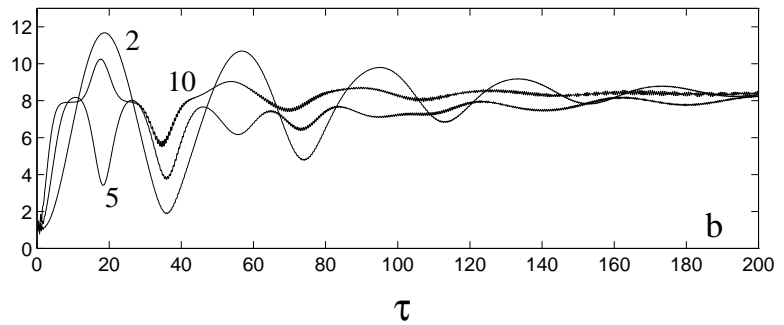

FIG. 4. The squeezing parameter $\xi$ versus the scaled time $\tau=g t$ for the initial coherent field state with $\bar{n}=36$ interacting with $N$ atoms $(N=2,5,10)$ prepared in (a) the fully-excited state $|j, j\rangle_{\text {at }}$ and (b) the ground state $|j,-j\rangle_{\text {at }}$.

As demonstrated in Fig. \#, this long-time behavior is fully correlated with the collapses and revivals of the Rabi oscillations. For even values of $N$, the squeezing parameter $\xi$ oscillates achieving its minima at integer multiples of $\tau_{R}$ and maxima at half-odd multiples of $\tau_{R}$. On the other hand, for odd values of $N$, we see that $\xi$ has minima at both half-odd and integer multiples of $\tau_{R}$. This difference between even and odd values of $N$ can be explained by the fact that squeezing depends on two-photon transitions, when a pair of photons is simultaneously absorbed or emitted by a pair of atoms. The oscillations of $\xi$ decay with time and abandon their regular form. This decay is correlated with the loss of regularity in the behavior of the atomic inversion, caused by the overlaps of neighboring revivals. The behavior of $\xi$ is rather similar for $m=j$ and $m=-j$, but in the latter case the decay of the oscillations and the irregularity occur earlier, as the revival time is shorter and the overlaps of revivals begin earlier. Also, for $m=-j$ the decay rate of the squeezing oscillations increases rapidly with $N$, while for $m=j$ the dependence of the decay rate on $N$ is less pronounced. Inspecting the minima of $\xi$ in Fig. A, we clearly see that their period becomes longer for $m=j$ and shorter for $m=-j$ as $N$ increases, in full accordance with the formula (3.5) for the revival time.

For $m= \pm j$, the minima of $\xi$ on the long-time scale are above the vacuum level, i.e., $\xi>1$. However, squeezing is achieved at short times, soon after the beginning of the interaction. In this region the squeezing parameter exhibits fast oscillations, with $\xi$ falling below 1. Therefore, for $m= \pm j$ we will focus on the behavior of squeezing on the short-time scale. 


\section{Squeezing on the short-time scale: The fully-excited state}

We first consider the case of the initial fully-excited atomic state $(m=j)$. Squeezing is achieved for $\tau<2$ and appears at shorter times for larger values of $N$.

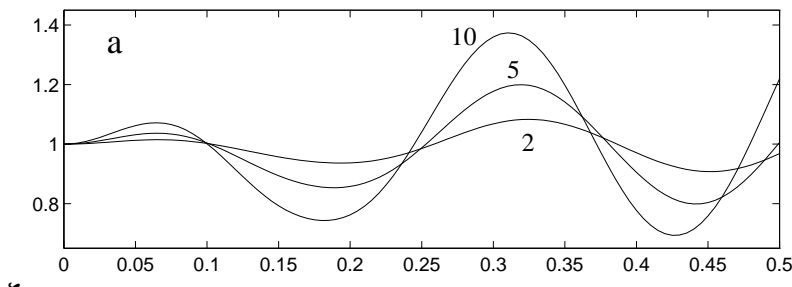

$\xi$

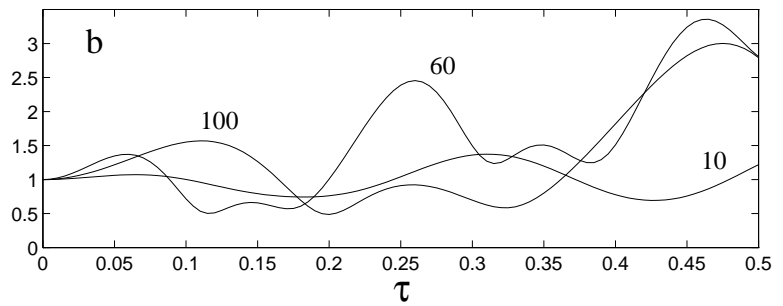

FIG. 5. The squeezing parameter $\xi$ versus the scaled time $\tau=g t$ for the initial coherent field state with $\bar{n}=36$ interacting with $N$ atoms prepared in the fully-excited state: (a) $N=2,5,10$, (b) $N=10,60,100$.

In Fig. 局 we see the short-time behavior of the squeezing parameter $\xi$ for $\bar{n}=36$ and various values of $N$. For relatively small values of $N(N \leq 10), \xi$ exhibits quite regular oscillations whose amplitude increases with $N$. However, for $N \sim \bar{n}$ and larger, the oscillations of $\xi$ become irregular. In Fig. 6 the short-time behavior of $\xi$ is shown for $N=14$ and various values of $\bar{n}$. The oscillations of $\xi$ become more regular as $\bar{n}$ increases.

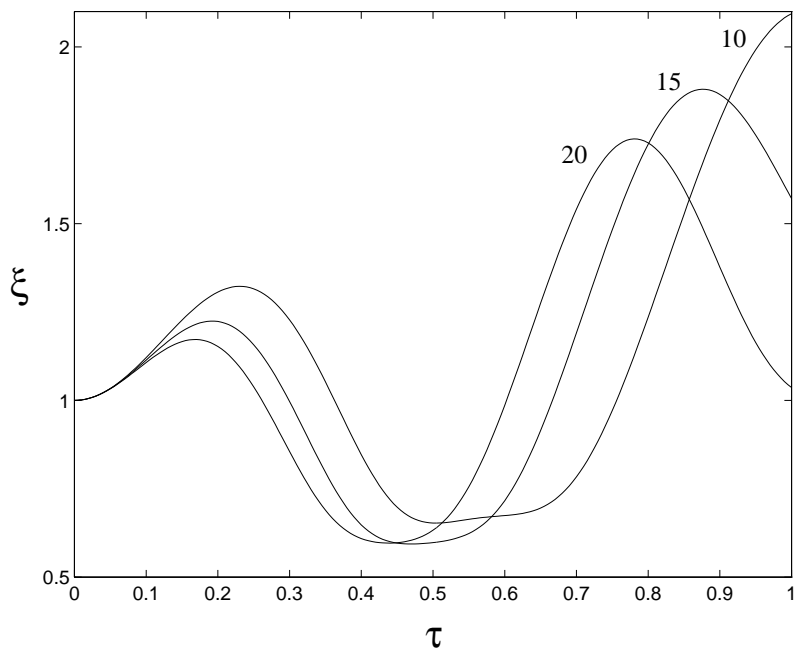

FIG. 6. The squeezing parameter $\xi$ versus the scaled time $\tau=g t$ for the initial coherent field state with $\bar{n}=10,15,20$ interacting with 14 atoms prepared in the fully-excited state.

It is interesting to investigate how the minimum value $\xi_{m}$ (i.e., the maximum of squeezing), achieved during the time evolution, depends on $N$ and $\bar{n}$.

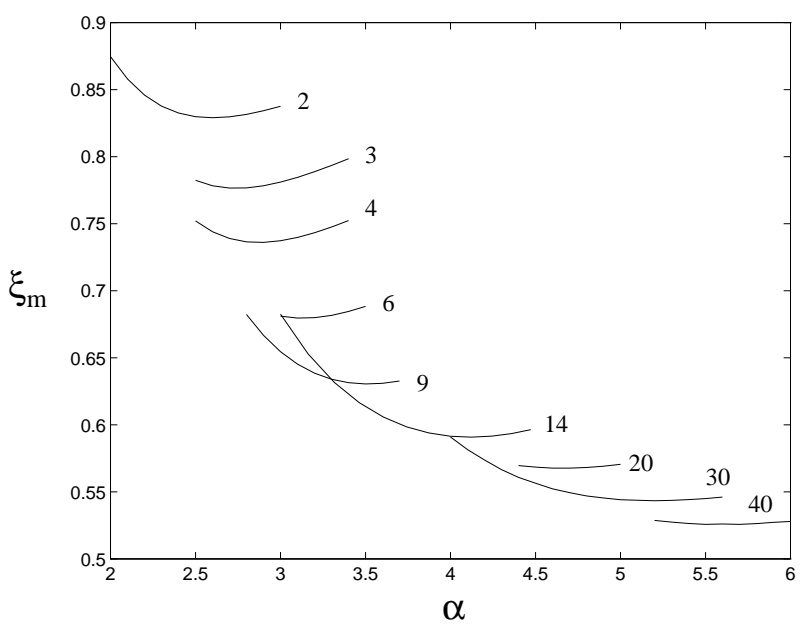

FIG. 7. The minimum value $\xi_{m}$ of the squeezing parameter versus the coherent amplitude $\alpha$ for various values of $N$.
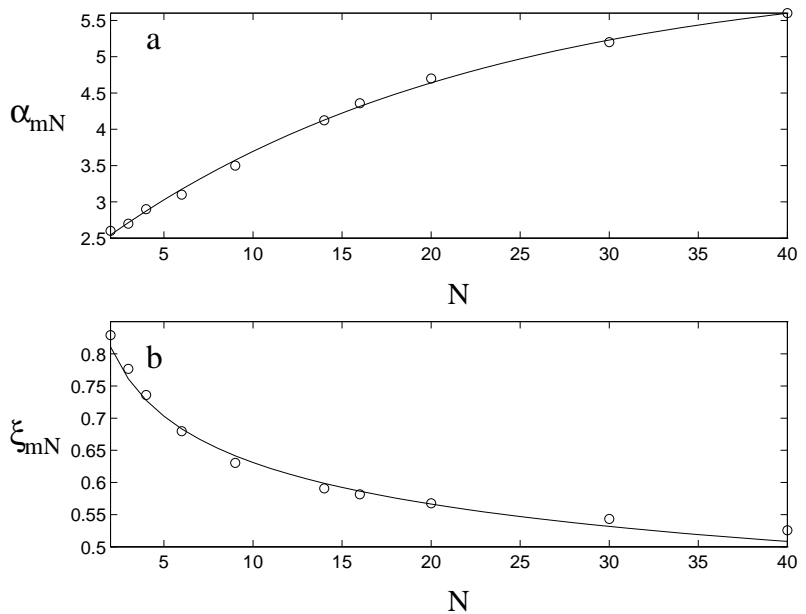

FIG. 8. (a) The value $\alpha_{m N}$ of the coherent amplitude, for which the squeezing parameter is minimized, versus $N$ : numerical results (circles) and empirical fitting of Eq. (4.2) (line); (b) the corresponding value $\xi_{m N}$ of the squeezing parameter versus $N$ : numerical results (circles) and empirical fitting of Eq. (4.3) (line).

We first consider the dependence of $\xi_{m}$ on $\alpha=\sqrt{\bar{n}}$ for given $N$. As shown in Fig. 7, $\xi_{m}$ has a minimum as a function of $\alpha$, i.e., for given $N$ there exists a value $\alpha_{m N}$ for which the minimum value $\xi_{m N}$ of the squeezing parameter is achieved. Figure 8 shows that $\alpha_{m N}$ increases and $\xi_{m N}$ decreases (squeezing improves) as $N$ increases. We found that the dependence of $\alpha_{m N}$ and $\xi_{m N}$ on $N$ at the considered range can be well approximated by the following empirical formulas:

$$
\begin{aligned}
& \alpha_{m N}=a-b e^{-c N}, \\
& \xi_{m N}=r N^{-s}
\end{aligned}
$$


where $a=6.21, b=4.03, c=0.0471, r=0.909, s=$ 0.156 .

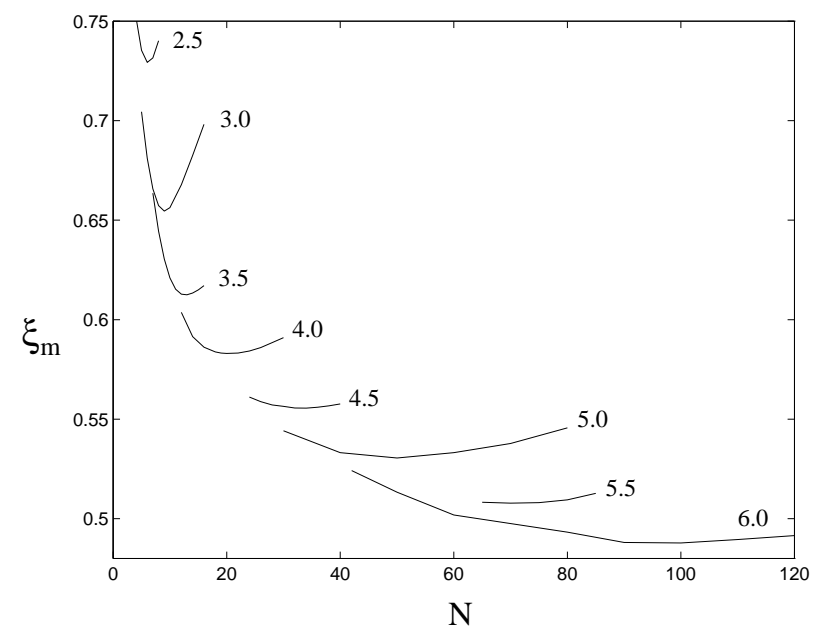

FIG. 9. The minimum value $\xi_{m}$ of the squeezing parameter versus $N$ for various values of $\alpha$.
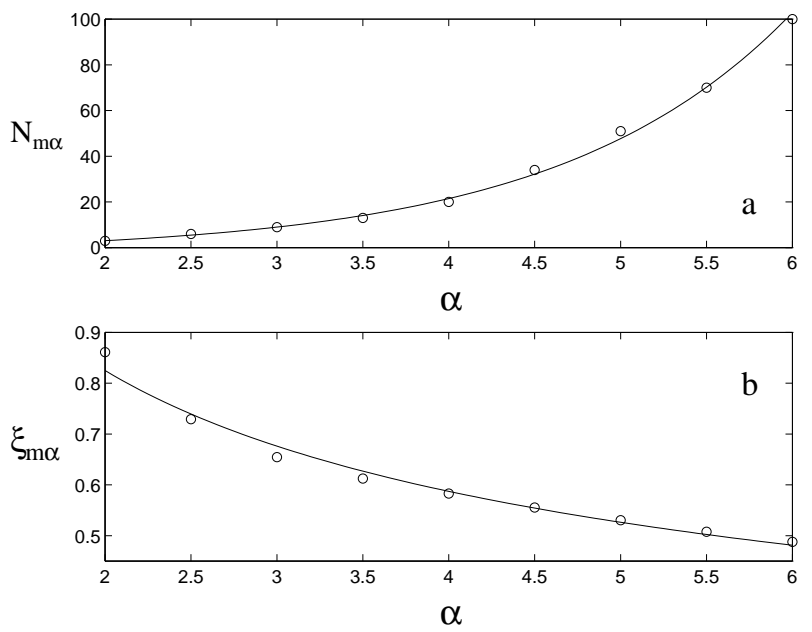

FIG. 10. (a) The value $N_{m \alpha}$ of the number of atoms, for which the squeezing parameter is minimized, versus $\alpha$ : numerical results (circles) and empirical fitting of Eq. (4.4) (line); (b) the corresponding value $\xi_{m \alpha}$ of the squeezing parameter versus $\alpha$ : numerical results (circles) and empirical fitting of Eq. (4.5) (line).

We next consider the dependence of $\xi_{m}$ on $N$ for given $\alpha$. As shown in Fig. 9, $\xi_{m}$ has a minimum as a function of $N$, i.e., for given $\alpha$ there exists a value $N_{m \alpha}$ for which the minimum value $\xi_{m \alpha}$ of the squeezing parameter is achieved. Figure 10 shows that $N_{m \alpha}$ increases and $\xi_{m \alpha}$ decreases (squeezing improves) as $\alpha$ increases. We found again that the dependence of $N_{m \alpha}$ and $\xi_{m \alpha}$ on $\alpha$ at the considered range can be well approximated by the following empirical formulas:

$$
\begin{aligned}
& N_{m \alpha}=-a+b e^{c \alpha}, \\
& \xi_{m \alpha}=r \alpha^{-s},
\end{aligned}
$$

where $a=2.30, b=1.216, c=0.743, r=1.159, s=$
0.490. Note that the relations $(4.2)$ and $(4.4)$ are not the inverse of each other. This fact can be easily understood, if one imagine $\xi_{m}$ as a two-dimensional function of $N$ and $\alpha$. When taking a section of $\xi_{m}$ along the $\alpha$ axis (i.e., for a given $N$ ), one will find the minimum for a certain value of $\alpha$. However, when fixing this value of $\alpha$ and going along the $N$ axis, a minimum will be found, in general, for a different $N$.

Finally, we would like to compare our numerical results with approximate analytical expressions derived by Retamal et al. [22] in the strong-field limit $\bar{n} \gg N$. They found 22 the following expression for the minimum of the squeezing parameter, achieved during the time evolution,

$$
\xi_{m}=\left(1-a \frac{N}{\alpha}+b \frac{N^{2}}{8 \alpha^{2}}\right)^{1 / 2}
$$

where $a=1 / \sqrt{e} \approx 0.606$ and $b=1+a-a^{2}-a^{4} \approx$ 1.103. Then, for given $\alpha$, the squeezing parameter will be minimized by

$$
N_{m \alpha}=\frac{4 a}{b} \alpha \approx 2.2 \alpha
$$

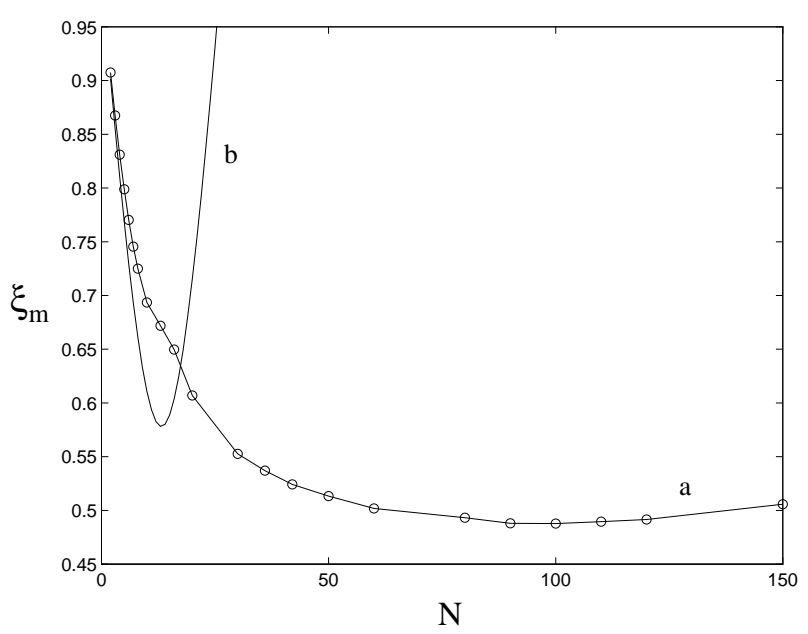

FIG. 11. The minimum value $\xi_{m}$ of the squeezing parameter versus $N$ for $\alpha=6$ : (a) numerical result, (b) approximate analytical formula $(4.6)$.

In Fig. 11 we compare our numerical results for $\xi_{m}$ with the approximate formula (4.6) for $\alpha=6(\bar{n}=36)$. A good agreement is found only for very small values of $N$, while the values of $N_{m \alpha}$ are absolutely different $\left(N_{m \alpha} \approx 100\right.$ for our numerical calculations and $N_{m \alpha} \approx$ 13 for the analytical approximation). This discrepancy can be explained by the fact that the numerical values of $N_{m \alpha}$ shown in Fig. 10 do not satisfy the strong-field condition $\bar{n} \gg N$. For the considered range of $\bar{n}(\leq$ 36 ), the approximate solution (4.6) is not valid. From Eq. (4.7) one can see that the strong-field condition will be satisfied for the optimal value $N_{m \alpha}$ when $N_{m \alpha} / \bar{n} \approx$ $2.2 / \alpha \ll 1$. This means that Eq. (4.7) gives a true value of $N_{m \alpha}$ only for $\bar{n} \sim 10^{4}$ or more. According to Eq. (4.6), 
the absolute minimum of the squeezing parameter that can be achieved in the strong-field limit is $\xi \approx 0.58$. For $\bar{n}=36$ (the maximum $\bar{n}$ we considered), the best value of squeezing is $\xi \approx 0.49$ and it is achieved for $N_{m \alpha} \approx 100$.

\section{Squeezing on the short-time scale: The ground state}

We next consider the case when the initial atomic state is the ground state $(m=-j)$. In this case we can distinguish three different regimes: the strong-field regime $(\bar{n} \gg N)$, the weak-field regime $(\bar{n} \ll N)$, and the intermediate regime $(\bar{n} \sim N)$.

The short-time behavior of the squeezing parameter $\xi$ in the strong-field regime is shown in Fig. 12 (for $\alpha=30$ and $N=2,6,16$ ). We see that $\xi$ exhibits fast regular oscillations, whose frequency is almost independent of $N$ (this is just the strong-field Rabi frequency $\Omega_{\bar{n},-j}^{(s)}=2 \sqrt{\bar{n}-N / 2+1 / 2}$, and the dependence on $N$ is very weak because $\bar{n}$ is very large), but the amplitude increases with $N$. Therefore, the minimum value $\xi_{m}$ of the squeezing parameter decreases as $N$ increases. A similar behavior is found also for the case $m=j$ in the strongfield regime. An interesting feature of this regime is that the minimum of $\xi$ is achieved after a relatively large number of oscillations (the same time for different values of $N$ ), while in the intermediate regime the minimum of $\xi$ is achieved, as a rule, in the first or second oscillation. As can be seen from Fig. 13, in the strong-field regime the decrease of $\xi_{m}$ is linear and can be well approximated by the empirical formula

$$
\xi_{m}=-f(\alpha) N+k,
$$

where the slope $f(\alpha)$ is a monotonically decreasing function of $\alpha$ and the free term $k \approx 1$ is independent of $\alpha$.

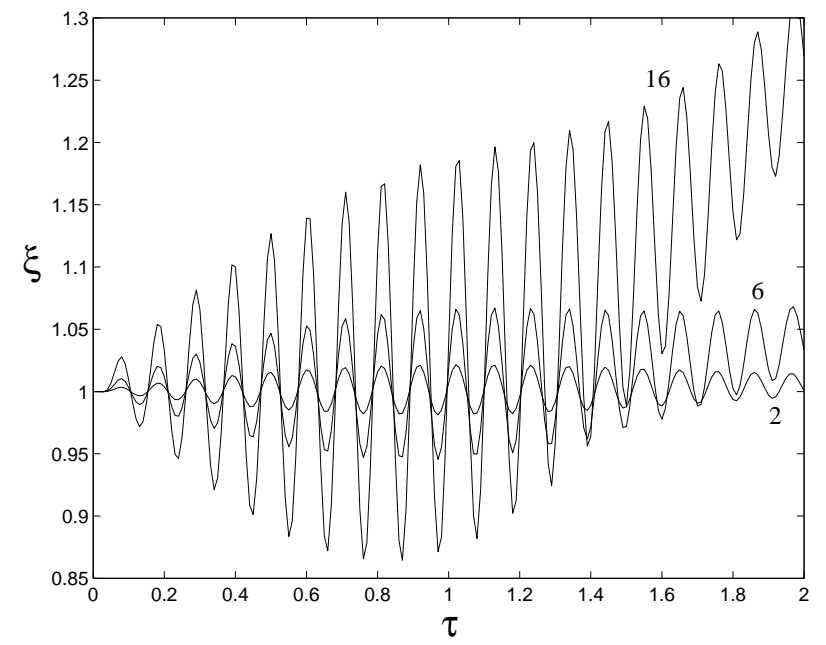

FIG. 12. The squeezing parameter $\xi$ versus the scaled time $\tau=g t$ for the initial coherent field state with $\alpha=30$ interacting with $N$ atoms $(N=2,6,16)$ prepared in the ground state.

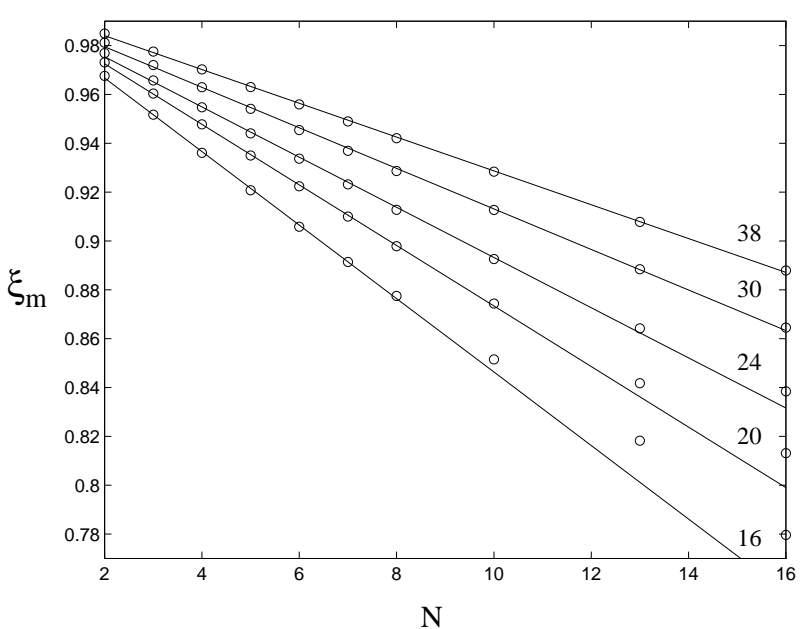

FIG. 13. The minimum value $\xi_{m}$ of the squeezing parameter versus $N$ for various values of $\alpha$ : numerical results (circles) and linear fitting of Eq. (4.8) (line). The fitting is good in the strong-field regime $\bar{n} \gg \bar{N}$.
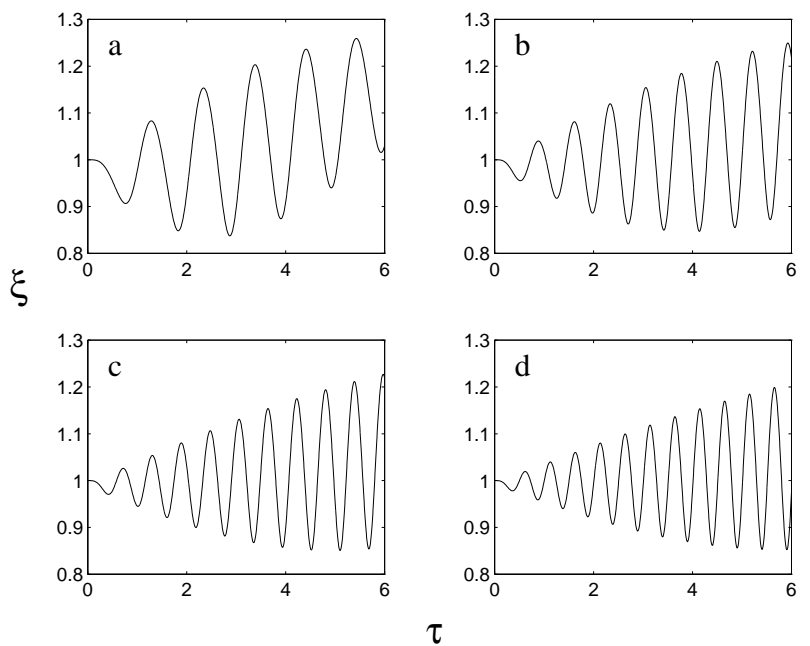

FIG. 14. The squeezing parameter $\xi$ versus the scaled time $\tau=g t$ for the initial coherent field state with $\alpha=1$ interacting with $N$ atoms prepared in the ground state: (a) $N=10$, (b) $N=20$, (c) $N=30$, (d) $N=40$.

It is well known 27,28,32,33 that in the weak-field regime the behavior of the system is very regular (note that for the case of the fully-excited initial state the weakfield regime actually does not exist). In Fig. 14 we see the evolution of the squeezing parameter $\xi$ for relatively short times (for $\alpha=1$ and $N=10,20,30,40$ ). The oscillations of $\xi$ are quite regular and their frequency increases with $N$ (this is just the weak-field Rabi frequency $\Omega_{\bar{n},-j}^{(w)}=$ $2 \sqrt{N-\bar{n} / 2+1 / 2}$, while the amplitude does not change significantly. The value of squeezing achieved in this limit is rather modest (as a rule, $\xi$ does not decrease below 0.85). A perturbative analytical approach to the Dicke model dynamics in the weak-field regime was developed by Kozierowski and co-workers 27, 28, 32,33 and used for the study of squeezing in Ref. [23]. 


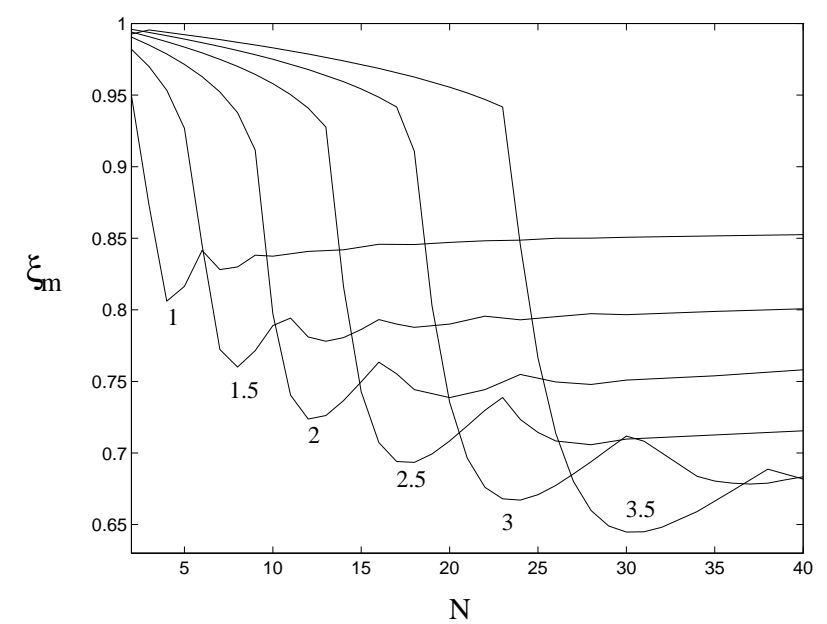

FIG. 15. The minimum value $\xi_{m}$ of the squeezing parameter versus $N$ for various values of $\alpha$.

We focus our attention on properties of squeezing in the intermediate regime, where no analytical approximation can be used. We study the minimum value $\xi_{m}$, achieved by the squeezing parameter during the time evolution, for $N$ in the range between 2 and 40 and for $\alpha$ between 1.0 and 6.5. The results are presented in Fig. 15, where $\xi_{m}$ is plotted versus $N$ for various values of $\alpha$. The typical behavior for given $\alpha$ is as follows. Initially, $\xi_{m}$ decreases slowly with $N$, but then steeply sinks down and acquires a minimum at a certain value of $N$. After the minimum, $\xi_{m}$ slightly oscillates and then saturates for large $N$ 's at an almost constant value. The region of linear decrease, occurring in the strong-field regime, appears only for $\alpha \geq 4$. This leads to an additional minimum at small values of $N$, but it is less pronounced than the main minimum at larger $N$ 's.
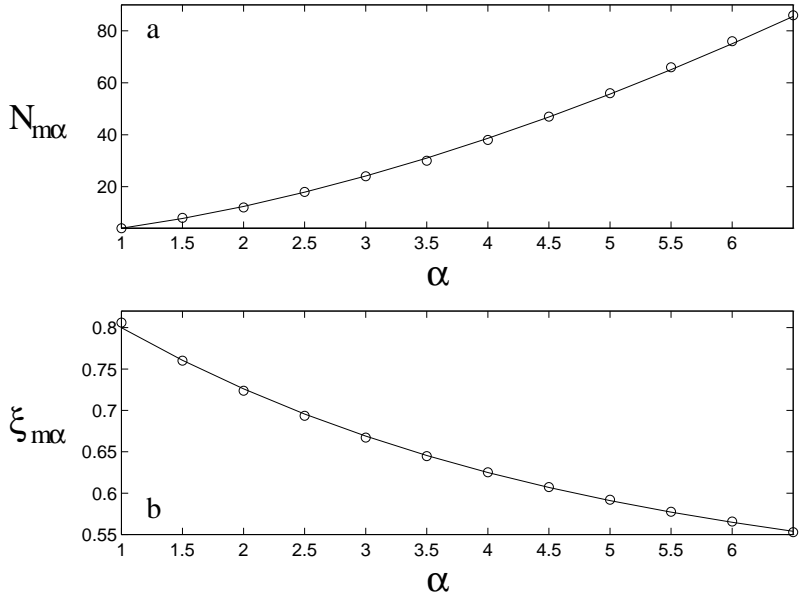

FIG. 16. (a) The value $N_{m \alpha}$ of the number of atoms, for which the squeezing parameter is minimized, versus $\alpha$ : numerical results (circles) and empirical fitting of Eq. (4.9) (line); (b) the corresponding value $\xi_{m \alpha}$ of the squeezing parameter versus $\alpha$ : numerical results (circles) and empirical fitting of Eq. (4.10) (line).
The value $N_{m \alpha}$, which gives optimal squeezing for given $\alpha$, and the corresponding minimum value $\xi_{m \alpha}$ of the squeezing parameter are shown in Fig. 16 as functions of $\alpha$. As $\alpha$ increases, squeezing improves ( $\xi_{m \alpha}$ decreases) and the minimum occurs at larger $N_{m \alpha}$. The dependence of $N_{m \alpha}$ and $\xi_{m \alpha}$ on $\alpha$ at the considered range can be well approximated by the following empirical formulas:

$$
\begin{aligned}
& N_{m \alpha}=k \alpha^{\gamma}, \\
& \xi_{m \alpha}=x+y e^{-z \alpha},
\end{aligned}
$$

where $k=1.382, \gamma=1.639, x=0.476, y=0.420, z=$ 0.259 . The largest value of $\alpha$ we considered is 6.5 . If we assume that Eq. (4.10) remains valid for arbitrarily large $\alpha$, then the absolute minimum of squeezing achievable with unexcited atoms is $\xi \approx 0.476$.

\section{B. Squeezing for the half-excited state and the factorization approximation}

Finally, we discuss the case when the initial atomic state is the half-excited Dicke state $(m=0)$. The temporal behavior of the squeezing parameter $\xi$ on the longtime scale is shown in Fig. 17. We see that there is a strong correlation between the collapses and revivals of the atomic inversion and the behavior of squeezing.

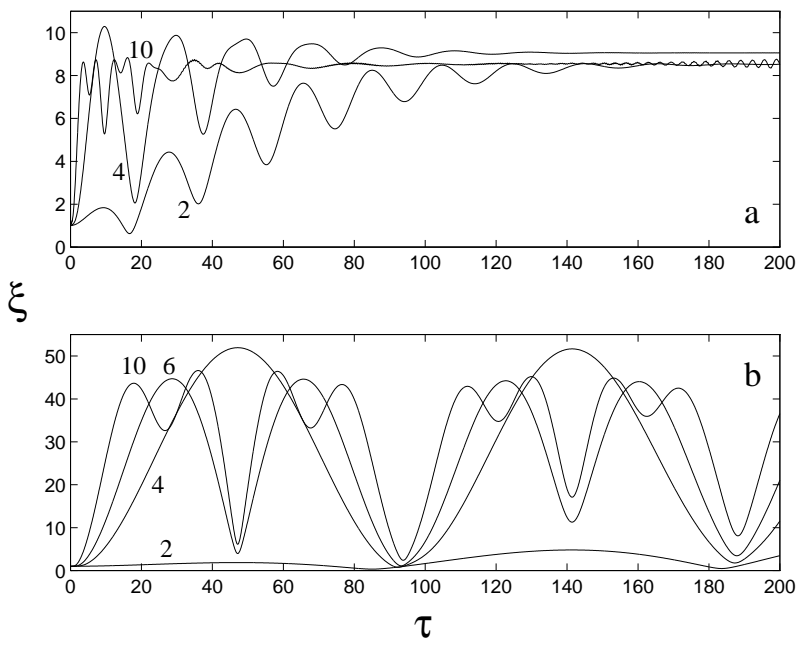

FIG. 17. The squeezing parameter $\xi$ versus the scaled time $\tau=g t$ for the initial coherent field state interacting with $N$ atoms prepared in the half-excited Dicke state: (a) $\bar{n}=36$, $N=2,4,10$, (b) $\bar{n}=900, N=2,4,6,10$.

For $\bar{n}=36$ and $N=2,4$, we see in Fig. 17)(a) that $\xi$ oscillates with a period of order $\tau_{R} / 2$ (recall that for $m=0$ we find relatively strong half-time revivals). However, after a number of periods, the amplitude of the oscillations decays and $\xi$ becomes nearly constant. This decay is correlated with the loss of regularity in the behavior of the atomic inversion that happens when neighboring revivals overlap. For larger numbers of atoms, the behavior of the atomic inversion is very irregular, and so is the behavior 
of squeezing. In particular, for $N=10$, the oscillations of $\xi$ decay already after $\tau_{R} / 2$. The phenomenon of the collapses and revivals is quite regular for very large values of $\bar{n}$. We see in Fig. 17.(b) that for $\bar{n}=900$ the squeezing parameter also behaves very regularly. The structure of the oscillations of $\xi$ becomes more complicated (but keeps the regularity) as $N$ increases. For all values of $N$, the squeezing parameter reaches deep minima at times just before integer multiples of $\tau_{R} / 2$. The larger the value of $N$, the closer is the minimum of $\xi$ to $\tau_{R} / 2$. (For $N \geq 6$, we also find additional minima of $\xi$, but they are not so deep.)

The phenomenon of field squeezing for the half-excited Dicke state is drastically different from what we found for the fully-excited and ground states. In the former case $(m=0)$, for moderate values of $\bar{n}(\alpha<30)$, the minima of $\xi$ decrease below 1 (i.e., squeezing occurs) only for $N=2$. This behavior is in contrast to the situation in the latter case $(m= \pm j)$, where squeezing can be achieved for any $N$ (with a proper choice of $\alpha$ ) and is, moreover, enhanced by increasing $N$. However, just two atoms prepared in the half-excited Dicke state can produce quite strong squeezing.

In Fig. 18 we see the temporal behavior of $\xi$ for $N=2$ and $\alpha=6,10,16,30$. The minima of $\xi$, which occur at times before integer multiples of $\tau_{R} / 2$, become deeper (i.e., squeezing improves) as $\bar{n}$ increases. It is very important to note that here squeezing is achieved at minima of the oscillations on the long-time scale. (Fast oscillations of $\xi$ on the short-time scale, which produce squeezing for $m= \pm j$, are negligible for the case $m=0$.) Consequently, for $m=0$ the duration of squeezing is essentially longer than for $m= \pm j$.

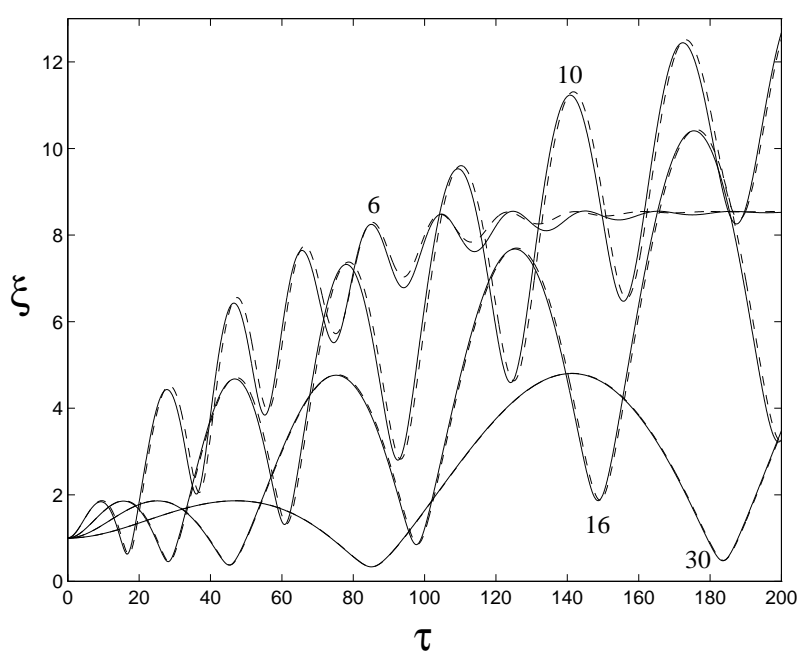

FIG. 18. The squeezing parameter $\xi$ versus the scaled time $\tau=g t$ for the initial coherent field state with $\alpha=6,10,16,30$ interacting with two atoms prepared in the half-excited Dicke state: numerical results (solid line) and the factorization approximation (dashed line).

Actually, this kind of squeezing on the long-time scale obtained for two atoms in the half-excited Dicke state has the same physical origin as revival-time squeezing in the Jaynes-Cummings model [17,18]. This long-time squeezing can be explained by means of the factorization approximation for the semiclassical atomic states [19]. This approximation is valid for a strong initial coherent field and for times short compared with $\tau_{0} \sim \bar{n}$.

In a semiclassical treatment, one replaces the boson operators of the field in the interaction Hamiltonian with $c$-numbers. The eigenstates $|p\rangle_{\text {at }}$ of this semiclassical Hamiltonian (called the semiclassical states) are just the eigenstates of the operator $J_{+}+J_{-}=2 J_{x}$ with the eigenvalues $\lambda_{p}=N-2 p(p=0,1, \ldots, N)$. An atomic state (and, in particular, a Dicke state) can be expanded in the basis of the semiclassical states. The factorization approximation means that if the atoms are prepared initially in a semiclassical state, then the total wave function of the system can be approximately written as a product of its field and atomic parts 19,11]:

$$
\left|\Psi_{p}(t)\right\rangle \approx\left|A_{p}(t)\right\rangle_{\text {at }} \otimes\left|\Phi_{p}(t)\right\rangle_{\mathrm{f}} .
$$

Using the factorization approximation, one can analytically estimate mean values of the field operators corresponding to a specific initial semiclassical atomic state $|p\rangle_{\text {at }}$. In particular, one obtains (as usual, we assume that $\alpha=\sqrt{\bar{n}}$ is real):

$$
\begin{aligned}
\langle a\rangle_{p}= & \alpha \exp \left[-\frac{i \lambda_{p} \tau}{2 \alpha}\left(1-\frac{1}{4 \alpha^{2}}\right)\right] \\
& \times \exp \left\{\alpha^{2}\left[e^{i \lambda_{p} \tau / 4 \alpha^{3}}-1-\frac{i \lambda_{p} \tau}{4 \alpha^{3}}\right]\right\}, \\
\left\langle a^{2}\right\rangle_{p}= & \alpha^{2} \exp \left[-\frac{i \lambda_{p} \tau}{2 \alpha}\left(2-\frac{1}{\alpha^{2}}\right)\right] \\
& \times \exp \left\{\alpha^{2}\left[e^{i \lambda_{p} \tau / 2 \alpha^{3}}-1-\frac{i \lambda_{p} \tau}{2 \alpha^{3}}\right]\right\} .
\end{aligned}
$$

Also, $\left\langle a^{\dagger}\right\rangle_{p}$ and $\left\langle a^{\dagger 2}\right\rangle_{p}$ are given by complex conjugates of $\langle a\rangle_{p}$ and $\left\langle a^{2}\right\rangle_{p}$, respectively, while $\left\langle a^{\dagger} a\right\rangle_{p}=\bar{n}$ can be taken constant. Then an approximate expression for the squeezing parameter can be easily obtained. For the semiclassical state $|p\rangle_{\text {at }}$, one finds [22]

$$
\begin{aligned}
\xi_{p}^{2}= & +\left[2 \bar{n}\left(e^{-T_{p}^{2} / 8}-e^{-T_{p}^{2} / 16}\right)\right. \\
& \left.+\frac{T_{p}^{2}}{16}\left(e^{-T_{p}^{2} / 16}-4 e^{-T_{p}^{2} / 8}\right)\right] \cos \left(\frac{\lambda_{p} \tau}{\sqrt{\bar{n}}}\right) \\
& +\frac{T_{p} \sqrt{\bar{n}}}{2}\left(2 e^{-T_{p}^{2} / 8}-e^{-T_{p}^{2} / 16}\right) \sin \left(\frac{\lambda_{p} \tau}{\sqrt{\bar{n}}}\right) \\
& +2 \bar{n}\left(1-e^{-T_{p}^{2} / 16}\right),
\end{aligned}
$$

where $T_{p}=\lambda_{p} \tau / \bar{n}$. It can be seen that squeezing is achieved for any semiclassical state, except for those with 
$\lambda_{p}=0$ (then $\xi_{p}=1$ is constant as long as the factorization approximation is valid). For $\lambda_{p} \neq 0$, the oscillations of the squeezing parameter achieve minima for times

$$
\tau_{\mathrm{sq}}^{(p)} \approx 0.9 \tau_{R}^{(p)}, 1.95 \tau_{R}^{(p)}, \ldots,
$$

where the revival time for the state $|p\rangle_{\text {at }}$ is given by

$$
\tau_{R}^{(p)}=\frac{2 \pi \sqrt{\bar{n}}}{\left|\lambda_{p}\right|} \approx \frac{\tau_{R}}{\left|\lambda_{p}\right|}
$$

The minima of $\xi_{p}$ become deeper (i.e., squeezing improves) as $\bar{n}$ increases.

Using formulas (4.12) and (4.13), one can calculate an approximate expression for the squeezing parameter for an initial atomic state which is a superposition of the semiclassical states. In particular, we will be interested in approximate results for squeezing behavior of the Dicke states. First, note that in the Jaynes-Cummings model $(N=1)$ there are just two semiclassical states with $\lambda_{p}= \pm 1$, whose squeezing behavior is the same, for a good degree of accuracy. Consequently, any initial atomic state in the Jaynes-Cummings model (and, in particular, the ground and excited states) will exhibit the same squeezing behavior (within the validity of the factorization approximation). These considerations explain the appearance of significant squeezing in the strong-field regime of the Jaynes-Cummings model for times near integer multiples of the revival time $\tau_{R}$.

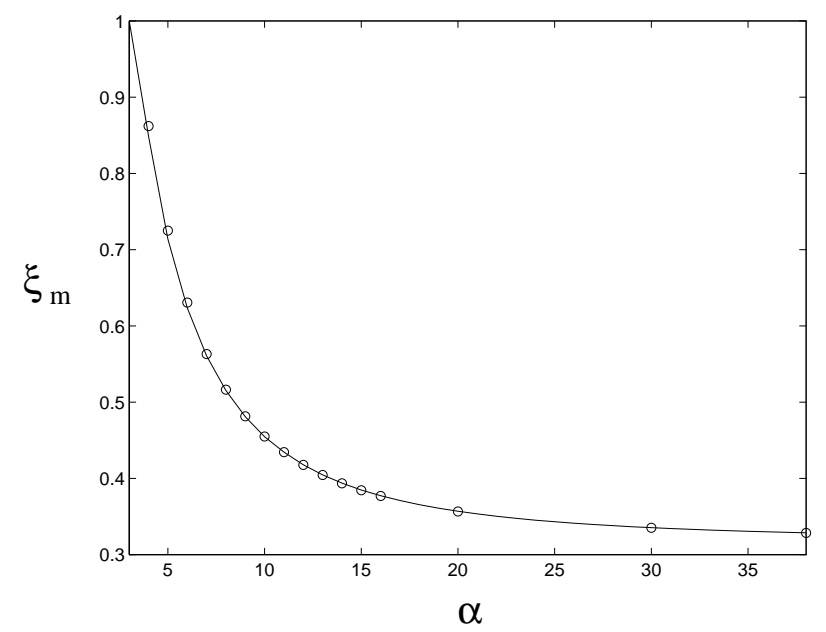

FIG. 19. The minimum value $\xi_{m}$ of the squeezing parameter versus the coherent amplitude $\alpha$ for two atoms prepared in the half-excited Dicke state: numerical results (circles) and the factorization approximation (line).

In the case of the Dicke model with two atoms, there are three semiclassical states; two of these states (with $\lambda_{p}= \pm 2$ ) lead to squeezing, while for the third state (with $\lambda_{p}=0$ ) no squeezing is found ( $\xi_{p}=1$ is constant). The half-excited Dicke state $|1,0\rangle$ is a superposition of the two semiclassical states with $\lambda_{p}= \pm 2$, while the fullyexcited and ground states also include the third semi- classical state with $\lambda_{p}=0$. Consequently, the squeezing behavior of the half-excited Dicke state is very close to the behavior of a semiclassical state with $\left|\lambda_{p}\right|=2$, while for the fully-excited and ground states squeezing is spoiled by the influence of the semiclassical state with $\lambda_{p}=0$. These considerations explain why squeezing on the long-time scale can be achieved only for the halfexcited Dicke state $|1,0\rangle$. The factorization approximation also explains the appearance of the minima of the squeezing parameter at times close to integer multiples of $\tau_{R} / 2$, whose depth increases with $\bar{n}$. From Fig. 18 we see that the factorization approximation describes very well the squeezing behavior of the two-atom half-excited Dicke state for large values of $\bar{n}$. As shown in Fig. 19, the minimum value $\xi_{m}$ of the squeezing parameter decreases monotonically with $\alpha$. For $\bar{n}>10$, the factorization approximation is in excellent agreement with the numerical results. Similar results are also obtained in the strongfield regime of the Jaynes-Cummings model. For $\alpha=38$ (the maximum value of $\alpha$ we considered), the best value of the squeezing parameter is 0.3285 . This is much better than the optimal values of squeezing which are obtained for the fully-excited or unexcited atoms when one takes $N \sim 10^{2}$.

For atom numbers $N \geq 3$, any Dicke state will be a superposition of the semiclassical states with different values of $\left|\lambda_{p}\right|$. Since the minima of the squeezing parameter $\xi_{p}$ occur at different times for different $\left|\lambda_{p}\right|$, squeezing on the long-time scale will be spoiled for the Dicke states of three and more atoms. It can be shown 22, that for a Dicke state of $N$-atom system the condition for the existence of squeezing in the revival regime is $\bar{n}>(2 N)^{4}$. Of course, the two-atom half-excited Dicke state is an exception to this rule, as it involves only two semiclassical states with the same value of $\left|\lambda_{p}\right|$. However, the above condition is in good agreement with our numerical results for $N \geq 3$.

As we see, the factorization approximation can be very useful for explaining many features of the field-atom interaction in the Dicke model. However, this approximation fails to predict some interesting phenomena found by using numerical calculations. In particular, squeezing on the short-time scale, which clearly dominates for the fully-excited and ground atomic states (especially, for large values of $N$ ), is not predicted by the factorization approximation. We also observe that the suppression of the revival amplitude for the half-excited Dicke state cannot be described within this approximation. This can be readily understood by recalling the exact expression (3.10) describing the evolution of the population inversion for the half-excited state $|1,0\rangle$. The amplitude of the Rabi oscillations here is of the order of $1 / \bar{n}$, which is neglected in the factorization approximation. 


\section{CONCLUSIONS}

In this paper we considered in detail properties of the system of $N$ two-level atoms interacting with a singlemode cavity field (the Dicke model). When the field is initially in the coherent state with a sufficiently large mean photon number, the dynamics of the system is quite regular, and the Rabi oscillations of the atomic inversion exhibit an interesting quantum phenomenon of collapses and revivals. We studied how this phenomenon is influenced by collective atomic effects. The main conclusion is that the role of the collective effects is determined by the initial atomic state. We found that by preparing just two atoms in the half-excited Dicke state one can cause greater effect on the behavior of the system than by collecting tens or even hundreds of excited or unexcited atoms. In the phenomenon of the collapses and revivals, the half-excited Dicke state causes two basic effects: the revival amplitude is strongly suppressed (analogously to the trapping phenomenon for a single atom in the equally weighted superposition state) and the revival time is halved. The two-atom half-excited Dicke state also leads to very interesting squeezing behavior. It is the only Dicke state for $N \geq 2$ which exhibits strong squeezing on the long-time scale, similarly to the behavior found in the strong-field regime of the Jaynes-Cummings model.

\section{ACKNOWLEDGMENTS}

G.R. and C.B. gratefully acknowledge the financial help from the Technion. A.M. was supported by the Fund for Promotion of Research at the Technion and by the Technion VPR Fund.

* E-mail: ramon@physics.technion.ac.il

$\dagger \quad$ E-mail: costya@physics.technion.ac.il

‡ E-mail: ady@physics.technion.ac.il

[1] R. Dicke, Phys. Rev. 93, 99 (1954).

[2] C. Leonardi, F. Persico, and G. Vetri, Riv. Nuovo Cimento 9, 1 (1986).

[3] M. Tavis and F. W. Cummings, Phys. Rev. 170, 379 (1968).

[4] D. F. Walls and R. Barakat, Phys. Rev. A 1, 446 (1970).

[5] E. T. Jaynes and F. W. Cummings, Proc. IEEE 51, 89 (1963).

[6] L. Knoll and D. G. Welsch, Prog. Quantum Electron. 16, 135 (1992).

[7] B. W. Shore and P. L. Knight, J. Mod. Opt. 40, 1195 (1993).

[8] J. H. Eberly, N. B. Narozhny, and J. J. SánchezMondragón, Phys. Rev. Lett. 44, 1323 (1980); N. B. Narozhny, J. J. Sánchez-Mondragón, and J. H. Eberly, Phys. Rev. A 23, 236 (1981).

[9] S. M. Barnett and P. L. Knight, Opt. Acta 31, 435 (1984); 31, 1203 (1984).
[10] G. Drobný and I. Jex, Opt. Commun. 102, 141 (1993).

[11] S. M. Chumakov, A. B. Klimov, and J. J. SánchezMondragón, Phys. Rev. A 49, 4972 (1994).

[12] S. M. Chumakov and M. Kozierowski, Quantum Semiclass. Opt. 8, 775 (1996).

[13] H. M. Castro-Beltran, S. M. Chumakov, and J. J. Sánchez-Mondragón, Opt. Commun. 129, 184 (1996).

[14] K. Zaheer and M. S. Zubairy, Phys. Rev. A 39, 2000 (1989).

[15] P. Meystre and M. S. Zubairy, Phys. Lett. 89A, 390 (1982).

[16] A. S. Shumovsky, Fam Le Kien, and E. I. Aliskenderov, Phys. Lett. A 124, 351 (1987).

[17] J. R. Kukliński and J. L. Madajczyk, Phys. Rev. A 37, 3175 (1988).

[18] C. W. Woods and J. Gea-Banacloche, J. Mod. Opt. 40, 2361 (1993).

[19] J. Gea-Banacloche, Phys. Rev. A 44, 5913 (1991).

[20] M. Butler and P. D. Drummond, Opt. Acta 33, 1 (1986).

[21] J. Seke, Quantum Semiclass. Opt. 7, 161 (1995); Physica A 213, 587 (1995); 240, 635 (1997).

[22] J. C. Retamal, C. Saavedra, A. B. Klimov, and S. M. Chumakov, Phys. Rev. A 55, 2413 (1997).

[23] M. Kozierowski and S. M. Chumakov, (unpublished).

[24] F.-L. Li, X.-S. Li, D. L. Lin, and T. F. George, Phys. Rev. A 41, 2712 (1990).

[25] D. J. Wineland, J. J. Bollinger, W. M. Itano, and D. J. Heinzen, Phys. Rev. A 50, 67 (1994).

[26] I. R. Senitzky, Phys. Rev. A 3, 421 (1971).

[27] M. Kozierowski, S. M. Chumakov, J. Światłowski, and A. A. Mamedov, Phys. Rev. A 46, 7220 (1992).

[28] M. Kozierowski, S. M. Chumakov, and A. A. Mamedov, J. Mod. Opt. 40, 453 (1993).

[29] R. Bonifacio and G. Preparata, Phys. Rev. A 2, 336 (1970).

[30] S. Kumar and C. L. Mehta, Phys. Rev. A 21, 1573 (1980); 24, 1460 (1981).

[31] J. Katriel and D. G. Hummer, J. Phys. A 14, 1211 (1981).

[32] M. Kozierowski, A. A. Mamedov, and S. M. Chumakov, Phys. Rev. A 42, 1762 (1990).

[33] M. Kozierowski, S. M. Chumakov, and A. A. Mamedov, Physica A 180, 435 (1992).

[34] G. J. Milburn, Opt. Acta 31, 671 (1984).

TABLE I. The ratio $f_{51}=h_{5} / h_{1}$, that represents the anharmonicity of the Hamiltonian eigenvalues, for $n=36$, $N=10,20,30$ and $m= \pm j, 0$.

\begin{tabular}{lcc}
\hline \hline$N$ & $m$ & $f_{51}$ \\
\hline 10 & $j$ & 5.0219 \\
20 & & 5.0184 \\
30 & & 5.0159 \\
\hline 10 & $-j$ & 5.0382 \\
20 & & 5.0638 \\
30 & & 5.1852 \\
\hline 10 & 0 & 5.0283 \\
20 & & 5.0309 \\
30 & & 5.0360 \\
\hline \hline
\end{tabular}

